H. Naito

Nagoya Math. J.

Vol. 129 (1993), 97-113

\title{
ON A LOCAL HÖLDER CONTINUITY FOR A MINIMIZER OF THE EXPONENTIAL ENERGY FUNCTIONAL
}

\author{
HISASHI NAITO ${ }^{\dagger}$
}

\section{Introduction}

Let $\Omega \subset \mathbf{R}^{m}$ be a bounded domain with smooth boundary, where $m \geq 2$. We consider the exponential energy functional

$$
E(u):=\int_{\Omega} e^{|\nabla u|^{2}} d x
$$

for $u: \Omega \rightarrow \mathbf{R}^{n}$, where $n \geq 2$.

For minima of certain functionals with suitable growth conditions, many authors have established regularity results [1,5,7,12]. For example, in [7], Hardt and Lin proved that mappings minimizing the $L^{p}$-norm of the gradient between compact Riemannian manifolds are smooth except singular sets with finite (dim $M-[p]-1)$-dimensional Hausdorff measure.

If a functional has sufficiently rapid growth, we can expect full regularity of minima. In fact, quite recently, Duc and Eells [2], Eells and Lemaire [3] show that, in the case of $n=1$, a minimizer $u$ of $E$ satisfies $u \in C^{\infty}(\Omega)$ for any smooth boundary data provided that $\Omega \subset \mathbf{R}^{m}$ is a strictly convex bounded domain.

In this paper, we consider a local regularity of minima of $E$ for the case of $n \geq 2$. Our main theorem is stated as follows:

(0.2) Theorem. For a given boundary data $g \in \cap_{1<p<\infty} W^{1-1 / p, p}\left(\partial \Omega, \mathbf{R}^{n}\right)$, there exists a unique minimizer of $E$ on the space $\left\{u \in \cap_{1<p<\infty} W^{1, p}\left(\Omega, \mathbf{R}^{n}\right):\left.u\right|_{\partial \Omega}=g\right\}$. If $B_{R}(a) \subset \Omega$, then there exists a number $\mu$ satisfying $0<\mu<1$ such that the minimizer belongs to $C^{1, \mu}\left(B_{R / 4}(a)\right)$.

Received October 25, 1991

$\dagger$ Partially supported by Yukawa Foundation and Japan Association for Mathematical Sciences. 
From now on, we abbreviate $W^{1-1 / p, p}\left(\partial \Omega, \mathbf{R}^{n}\right), W^{p}\left(\Omega, \mathbf{R}^{n}\right)$ to $W^{1-1 / p, p}(\partial \Omega)$, $W^{p}(\Omega)$, respectively.

For the sake of simplicity, we also use the following notations of function spaces. Let $W$ be the Banach space defined by

$$
W:=\bigcap_{1<p<\infty} W^{1, p}(\Omega),
$$

and the space of traces of elements of $W$ :

$$
B:=\bigcap_{1<p<\infty} W^{1-1 / p, p}(\partial \Omega) .
$$

For a given $g \in B$, the function space on which we consider the functional $E$ is denoted by

$$
W_{g}:=\left\{u \in W:\left.u\right|_{\partial \Omega}=g\right\}
$$

Moreover, to prove Theorem, we consider a Hilbert space

$$
X_{g}:=\left\{u \in H^{1}(\Omega):\left.u\right|_{\partial \Omega}=g\right\}
$$

for a given $g \in H^{1 / 2}(\Omega)$.

In this paper, $B_{r}(a)$ expresses the ball centered at $a \in \Omega$ with radius $r$, and we abbreviate $B_{r}:=B_{r}(a)$ when there is no confusion. Moreover $d$ stands for the distance from the center $a$ to $\partial \Omega$, i.e, $d:=\operatorname{dist}(a, \partial \Omega)$.

\section{The existence of a minimizer}

The aim of this section is to prove the following theorem.

(1.1) Theorem. For a given boundary data $g \in B$, there exists a unique minimizer of the functional $E$ on $W_{g}$.

The proof of Theorem 1.1 is divided into the following propositions.

First, we prove an existence and uniqueness theorem of minima on the space $X_{g}$

(1.2) Proposition. For a given boundary data $g \in B$, there exists a unique minimizer of the functional $E$ on $X_{g}$.

Proof. We note that the set $X_{g}$ is a closed convex subspace in $H^{1}(\Omega)$ and the functional $E$ is strongly convex and strongly lower semi-continuous on $X_{g}$. Therefore, $E$ is weakly lower semi-continuous on $X_{g}$. 
Moreover, we show that the functional $E$ is coercive on $X_{g}$. Namely, any sequence $\left\{u_{n}\right\}$ in $X_{g}$ which satisfies $\left\|u_{n}\right\| \rightarrow \infty$ contains a subsequence $\left\{u_{n}^{\prime}\right\}$ such that $E\left(u_{n}^{\prime}\right) \rightarrow \infty$. Let $\left\{u_{n}\right\} \subset X_{g}$ be a sequence which satisfies $\left\|u_{n}\right\| \rightarrow \infty$. Since $\Omega$ is bounded, using Poincaré's inequality, we see $\left\|\nabla u_{n}\right\|_{L^{2}(\Omega)} \rightarrow \infty$. On the other hand, for any $u \in X_{g}$, we have

$$
E(u)=\int_{\Omega} e^{|\nabla u|^{2}} d x \geq \int_{\Omega} d x+\frac{1}{2} \int_{\Omega}|\nabla u|^{2} d x .
$$

Therefore we get $E\left(u_{n}\right) \rightarrow \infty$.

Since $E$ is weakly lower semi-continuous, strongly convex and coercive, there exists a unique minimizer on $X_{g}$, (cf. [8]).

Hereafter we denote the minimizer of $E$ in $X_{g}$ by $u_{X}$.

(1.4) Proposition. The minimizer $u_{X}$ of $E$ in $X_{g}$ belongs to $W_{g}$, provided $g \in B$.

Proof. Since $E\left(u_{X}\right)<\infty$, we obtain

$$
\infty>E\left(u_{X}\right)=\int_{\Omega} e^{\left|\nabla u_{X}\right|^{2}} d x \geq \sum_{j=0}^{k} \frac{1}{(2 j) !} \int_{\Omega}\left|\nabla u_{X}\right|^{2 \jmath} d x
$$

Therefore we have $u_{X} \in W^{1,2 \jmath}(\Omega)$ for $j=2,3,4, \cdots$. Since $\left.u_{X}\right|_{\partial \Omega}=g \in B$, we get $u_{X} \in W_{g}$.

(1.5) Proposition. The minimizer $u_{X}$ of $E$ in $X_{g}$ is unique in $W_{g}$.

Proof. First we note that $B \subset H^{1 / 2}(\partial \Omega)$. If $g \in B$, then it is easy to see that $X_{g} \supset W_{g}$. Therefore we have

$$
\inf _{W_{g}} E(u) \geq \inf _{X_{g}} E(u) .
$$

Moreover since $u_{X} \in W_{g}$, we have

$$
E\left(u_{X}\right)=\inf _{W_{g}} E(u) .
$$

This equality implies that $u_{X}$ is a minimizer of $E$ in $W_{g}$. Since the functional $E$ is strongly convex and $W_{g}$ is convex, the proof of Proposition 1.4 shows the uniqueness of the minimizer. 


\section{Gradient bound}

In the following sections, we prove the regularity of a minimizer of $E$ by Moser's iteration method. To do so, we first have to prove that a minimizer of $E$ satisfies the Euler-Lagrange equation of the functional $E$, since the tangent space of the space $W$ does not coincide with itself. Proposition 2.1 is found in [3], but we describe the proof of it for the readers' convenience.

(2.1) Proposition. The minimizer of the functional $E$ in the class $W_{g}$ satisfies the Euler-Lagrange equation of $E$ weakly.

Proof. Let $e(\cdot)$ be the energy density of the functional $E$, i.e, $e(u)=e^{|\nabla u|^{2}}$. For $\phi \in W$ with $E(\phi)<\infty$, set

$$
f(t, x):=\frac{e(u+t(\phi-u))-e(u)}{t} \text { for } 0 \leq t \leq 1 .
$$

Since $e(\phi) \geq 1$ for any $\phi$, we have

$$
\text { (2.2) } 0 \leq \int_{\Omega} f(t, x) d x \leq t \int_{\Omega} e^{|\nabla \psi|^{2}} d x+(1-t) \int_{\Omega} e^{|\nabla u|^{2}} d x \quad \text { for } 0 \leq t \leq 1 .
$$

Moreover, since $e(\cdot)$ is convex, we have

$$
\begin{aligned}
& 0 \leq \frac{\partial f(t, x)}{\partial t}, \\
& 0 \leq f(t, x) \text { for } 0 \leq t \leq 1 .
\end{aligned}
$$

Therefore, by (2.2) and (2.3), $\lim _{t \downarrow 0} f(t, x)$ exists and we have

$$
\begin{array}{r}
\lim _{t \downarrow 0} f(t, x)=\nabla_{i} u^{\alpha} e^{|\nabla u|^{2}}\left(\nabla_{i} \phi^{\alpha}-\nabla_{i} u^{\alpha}\right) \in L^{1}, \\
\int_{\Omega} \nabla_{i} u^{\alpha} e^{|\nabla u|^{2}}\left(\nabla_{i} \phi^{\alpha}-\nabla_{i} u^{\alpha}\right) d x \geq 0 .
\end{array}
$$

Now we can replace $\phi$ to $\phi+s \varphi$ for any $s \in \mathbf{R}$ and any $\varphi \in C_{0}^{\infty}\left(\Omega, \mathbf{R}^{n}\right)$ in (2.4). Letting $s \rightarrow \infty$ or $s \rightarrow-\infty$, we have

$$
\int_{\Omega} \nabla_{i} u^{\alpha} \nabla_{i} \varphi^{\alpha} e^{|\nabla u|^{2}} d x=0
$$

for any $\varphi \in C_{0}^{\infty}\left(\Omega, \mathbf{R}^{n}\right)$. 
In the followings, we shall prove a bound of the gradient of minimizers. Since it will be proved by Moser's iteration method, we shall first describe the proof in the case of $m \geq 3$, for the sake of simplicity. After the completion of the proof in that case, we shall make a remark about the proof in the case of $m=2$.

In order to justify the calculation in the proof, it is sufficient to have

$$
\nabla_{i} u^{\alpha} e^{\frac{\gamma}{2}|\nabla u|^{2}} \in W_{\mathrm{loc}}^{1,2}(\Omega) \text { for } \gamma>-1
$$

(2.5) Proposition. Assume $m \geq 3$. Let $u$ be a minimizer of $E$. If $B_{r}(a) \subset \Omega$, then we have

$$
\nabla_{i} u^{\alpha} e^{\frac{\gamma}{2}|\nabla u|^{2}} \in W^{1,2}\left(B_{r}(a)\right) \text { for } \quad \gamma>-1 \text {. }
$$

Proof. Let $\Delta_{h} \varphi$ be the difference quotient of a function $\varphi$ in the direction $e_{k}$. Since $u$ is a minimizer, $u$ satisfies the Euler-Lagrange equation of $E$ weakly:

$$
\int_{\Omega} \nabla_{i} u^{\alpha} \nabla_{i} \varphi^{\alpha} e^{|\nabla u|^{2}} d x=0 \text { for all } \varphi \in C_{0}^{\infty}(\Omega)
$$

In (2.6), replace $\varphi$ to $\Delta_{-h} \varphi$, and substitute $\varphi^{\alpha}:=\Delta_{h} u^{\alpha} \eta^{2}$. Then we have

$$
\int_{\Omega} \Delta_{h}\left(\nabla_{i} u^{\alpha} e^{|\nabla u|^{2}}\right) \nabla_{i}\left(\Delta_{h} u^{\alpha} \eta^{2}\right) d x=0
$$

Using $\left.\left.|\nabla| \Delta_{h} u\right|^{2}\right|^{2}=\left.\left.\left|\Delta_{h}\right| \nabla u\right|^{2}\right|^{2}$ and Hölder's inequality, we have

$$
\begin{gathered}
\int_{\Omega}\left(\left|\Delta_{h} \nabla u\right|^{2}+\left.\left.\frac{1}{2}\left|\Delta_{h}\right| \nabla u\right|^{2}\right|^{2}\right) e^{|\nabla u|^{2}} \eta^{2} d x \\
\leq C \int_{\Omega}\left(\left|\Delta_{h} \nabla u\right|\left|\Delta_{h} u\right|+\left.|\nabla u|\left|\Delta_{h} u\right||\Delta h| \nabla u\right|^{2} \mid\right)|\nabla \eta| \eta e^{|\nabla u|^{2}} d x .
\end{gathered}
$$

Therefore we have

$$
\begin{gathered}
\int_{\Omega}\left(\left|\Delta_{h} \nabla u\right|^{2}+\left.\left.\frac{1}{2}\left|\Delta_{h}\right| \nabla u\right|^{2}\right|^{2}\right) e^{|\nabla u|^{2}} \eta^{2} d x \\
\leq C \int_{\Omega}\left(\left|\Delta_{h} \nabla u\right|^{2}+|\nabla u|^{2}\left|\Delta_{h} u\right|^{2}\right)|\nabla \eta|^{2} e^{|\nabla u|^{2}} d x .
\end{gathered}
$$

On the other hand, since $u^{\alpha}\left|\Delta_{h} u\right|^{2} \eta^{2} \in W_{0}^{1,2}(\Omega)$, and $u$ is a minimizer of $E$, we obtain

$$
\int_{\Omega} \nabla_{i} u^{\alpha} \nabla_{i}\left(u^{\alpha}\left|\Delta_{h} u\right|^{2} \eta^{2}\right) e^{|\nabla u|^{2}} d x=0
$$


Therefore we have

$$
\begin{aligned}
& \int_{\Omega}|\nabla u|^{2}\left|\Delta_{h} u\right|^{2} e^{|\nabla u|^{2}} \eta^{2} d x \\
& \leq\left.\int_{\Omega}|\nabla u||\nabla| \Delta_{h} u\right|^{2}|| u \mid e^{|\nabla u|^{2}} \eta^{2} d x \\
& \quad+2 \int_{\Omega}|\nabla u|\left|\Delta_{h} u\right|^{2}|u| e^{|\nabla u|^{2}}|\nabla \eta| \eta d x \\
& \leq\left.\left.\varepsilon \int_{\Omega}|\nabla| \Delta_{h} u\right|^{2}\right|^{2} e^{|\nabla u|^{2}} \eta^{2} d x+C \sup _{\Omega}|u| \int_{\Omega}|\nabla u|^{2} e^{|\nabla u|^{2}} \eta^{2} d x \\
& \quad+\varepsilon \int_{\Omega}|\nabla u|^{2}\left|\Delta_{h} u\right|^{2} e^{|\nabla u|^{2}} \eta^{2} d x+C \sup _{\Omega}|u| \int_{\Omega}\left|\Delta_{h} u\right|^{2} e^{|\nabla u|^{2}} \eta^{2} d x .
\end{aligned}
$$

Hence we obtain

$$
\begin{aligned}
& \int_{\Omega}|\nabla u|^{2}\left|\Delta_{h} u\right|^{2} e^{|\nabla u|^{2}} \eta^{2} d x \\
& \leq\left.\left.\varepsilon \int_{\Omega}|\nabla| \Delta_{h} u\right|^{2}\right|^{2} e^{|\nabla u|^{2}} \eta^{2} d x \\
& \quad+C \sup _{\Omega}|u| \int_{\Omega}\left(|\nabla u|^{2}+\left|\Delta_{h} u\right|^{2}\right) e^{|\nabla u|^{2}} \eta^{2} d x .
\end{aligned}
$$

Combining (2.7) and (2.8), we obtain

$$
\begin{aligned}
& \int_{\Omega}\left(\left|\nabla \Delta_{h} u\right|^{2}+\left.\left.\left|\Delta_{h}\right| \nabla u\right|^{2}\right|^{2}\right) e^{|\nabla u|^{2}} \eta^{2} d x \\
\leq & C r^{-4} \int_{\Omega}\left(\left|\Delta_{h} u\right|^{2}+|\nabla u|^{2}\right) e^{|\nabla u|^{2}} d x .
\end{aligned}
$$

On the other hand, we have

$$
\int_{\Omega}|\nabla u|^{2} e^{|\nabla u|^{2}} \eta^{2} d x \leq C r^{-2} E_{0} \sup _{\Omega}|u|,
$$

by putting $u^{\alpha} \eta \in W_{0}^{1,2}(\Omega)$ as a test function. Therefore we conclude that

$$
\int_{\Omega}\left(\left|\nabla \Delta_{h} u\right|^{2}+\left.\left.\left|\Delta_{h}\right| \nabla u\right|^{2}\right|^{2}\right) e^{|\nabla u|^{2}} \eta^{2} d x \leq C E_{0} .
$$

To show $\nabla u e^{\frac{\gamma}{2}|\nabla u|^{2}} \eta^{2} \in W_{0}^{1,2}(\Omega)$ for $-1<\gamma<0$, it is sufficient to show

$$
\int_{\Omega}\left(\left|\nabla^{2} u\right|^{2}+\left.\left.|\nabla u|^{2}|\nabla| \nabla u\right|^{2}\right|^{2}\right) d x<C
$$


Therefore, by (2.9), the claim follows.

(2.10) Proposition. Assume $m \geq 3$. Let $u$ be a minimizer of $E$. If $B_{r}(a) \subset \Omega$, then there exists a positive number $\varepsilon_{0}$ depending only on $m$ such that

$$
\left(r^{-m} \int_{B_{\gamma / 2}(a)} e^{(1+\varepsilon)|\nabla u|^{2}} d x\right)^{\frac{m-2}{m}} \leq C r^{-m} E_{0}
$$

for all $0<\varepsilon \leq \varepsilon_{0}$, where $C=C(m, \varepsilon)$ and $E_{0}=\int_{\Omega} e^{|\nabla u|^{2}} d x$.

Proof. First we take a negative number $\gamma$ as $\gamma>\max \{-1,-4 / m\}$. In (2.6), replace $\varphi$ to $\nabla_{k} \varphi$, set $w:=e^{|\nabla u|^{2}}$ and substitute $\varphi:=\nabla_{k} u^{\alpha} w^{\gamma / 2} \eta^{2}$. Then we have

$$
\begin{aligned}
0= & \int_{B_{r}} \nabla_{k}\left(\nabla_{i} u^{\alpha} e^{|\nabla u|^{2}}\right) \nabla_{i}\left(\nabla_{k} u^{\alpha} w^{\gamma / 2} \eta^{2}\right) d x \\
= & \int_{B_{r}}\left(\nabla_{k} \nabla_{i} u^{\alpha}+\nabla_{i} u^{\alpha} \nabla_{k}|\nabla u|^{2}\right) \nabla_{k} \nabla_{i} u^{\alpha} w^{\gamma / 2+1} \eta^{2} d x \\
& +\frac{\gamma}{2} \int_{B_{r}}\left(\nabla_{k} \nabla_{i} u^{\alpha}+\nabla_{i} u^{\alpha} \nabla_{k}|\nabla u|^{2}\right) \nabla_{k} u^{\alpha} w^{\gamma / 2+1} \nabla_{i}|\nabla u|^{2} \eta^{2} d x \\
& +2 \int_{B_{r}}\left(\nabla_{k} \nabla_{i} u^{\alpha}+\nabla_{i} u^{\alpha} \nabla_{k}|\nabla u|^{2}\right) \nabla_{k} u^{\alpha} w^{r / 2+1} \eta \nabla_{i} \eta d x \\
= & \text { (I) }+ \text { (II) }+ \text { (III). }
\end{aligned}
$$

By elementary calculations, the first term of the right hand side of (2.11) is equal to

$$
\text { (I) }=\int_{B_{r}}|\nabla \nabla u|^{2} w^{\gamma / 2+1} \eta^{2} d x+\left.\left.\frac{1}{2} \int_{B_{r}}|\nabla| \nabla u\right|^{2}\right|^{2} w^{\gamma / 2+1} \eta^{2} d x
$$

and the second term is equal to

$$
\text { (II) }=\left.\left.\frac{\gamma}{4} \int_{B_{r}}|\nabla| \nabla u\right|^{2}\right|^{2} w^{\gamma / 2+1} d x+\frac{\gamma}{2} \int_{B_{r}}\left|\left\langle\nabla u, \nabla|\nabla u|^{2}\right\rangle\right|^{2} w^{\gamma / 2+1} d x
$$

where $\langle\cdot, \cdot\rangle$ is the inner product $\mathbf{R}^{n}$. Since $-1<\gamma<0$, the sum of (2.12) and (2.13) is estimated by

$$
\begin{aligned}
(\mathrm{I}+\mathrm{II})= & \int_{B_{r}}\left(|\nabla \nabla u|^{2}+\frac{\gamma}{2}\left|\left\langle\nabla u, \nabla|\nabla u|^{2}\right\rangle\right|^{2}\right) w^{\gamma / 2+1} \eta^{2} d x \\
& +\left.\left.\frac{1}{2}\left(1+\frac{\gamma}{2}\right) \int_{B_{r}}|\nabla| \nabla u\right|^{2}\right|^{2} w^{\gamma / 2+1} \eta^{2} d x
\end{aligned}
$$




$$
\geq\left.\left.\frac{1}{2}\left(1+\frac{\gamma}{2}\right) \int_{B_{r}}|\nabla| \nabla u\right|^{2}\right|^{2} w^{\gamma / 2+1} \eta^{2} d x
$$

On the other hand, the third term is estimated from above by

$$
\begin{aligned}
\text { (III) } & \leq\left. C \int_{B_{r}}\left(1+|\nabla u|^{2}\right)|\nabla| \nabla u\right|^{2}|| \nabla \eta \mid \eta w^{\gamma / 2+1} d x \\
& \leq\left.\frac{C}{\delta} \int_{B_{r}}|\nabla| \nabla u\right|^{2}|| \nabla \eta \mid \eta w^{\gamma / 2+1+\delta} d x,
\end{aligned}
$$

for any $\delta>0$, since $x e^{x} \leq \frac{1}{\delta} e^{(1+\delta) x}$ for any $\delta>0$ and $x \geq 0$.

Inequalities (2.14) and (2.15) then give that

$$
\begin{aligned}
& \left.\left.\frac{1}{2}\left(1+\frac{\gamma}{2}\right) \int_{B_{r}}|\nabla| \nabla u\right|^{2}\right|^{2} w^{\gamma / 2+1} \eta^{2} d x \\
\leq & \left.\frac{C}{\delta} \int_{B_{r}}|\nabla| \nabla u\right|^{2}|\nabla \eta| \eta w^{\gamma / 2+1+\delta} d x .
\end{aligned}
$$

Since the left hand side and the right hand side of (2.16) are equal to

$$
\begin{aligned}
& (\mathrm{LHS})=\frac{2}{(1+\gamma / 2)} \int_{B_{r}}\left|\nabla\left(w^{\frac{1}{2}(\gamma / 2+1)}\right)\right|^{2} \eta^{2} d x, \\
& (\mathrm{RHS})=\frac{C}{\delta} \frac{2}{(1+\gamma / 2)} \int_{B_{r}}\left|\nabla\left(w^{\frac{1}{2}(\gamma / 2+1)}\right)\right| \eta w^{\frac{1}{2}(\gamma / 2+1)+\delta}|\nabla \eta| d x,
\end{aligned}
$$

and $w \geq 1$ and $-1<\gamma<0$, by taking $\delta=-\gamma / 4>0$ and the Hölder's inequality, we obtain

$$
\begin{aligned}
\int_{B_{\gamma}}\left|\nabla\left(w^{\frac{1}{2}(\gamma / 2+1)}\right)\right|^{2} \eta^{2} d x \leq C & \frac{2 \delta^{\prime}}{-\gamma} \int_{B_{\gamma}}\left|\nabla\left(w^{\frac{1}{2}(\gamma / 2+1)}\right)\right|^{2} \eta^{2} d x \\
& +C \frac{2}{-\gamma \delta^{\prime}} \int_{B_{r}} w^{\gamma / 4+1}|\nabla \eta|^{2} d x,
\end{aligned}
$$

for any $\delta^{\prime}>0$. Therefore, by taking $\delta^{\prime}=-\frac{\gamma}{4 C}$, there exists a positive constant $C=C(m, \gamma)$ such that

$$
\int_{B_{r}}\left|\nabla\left(w^{\frac{1}{2}(\gamma / 2+1)}\right)\right|^{2} \eta^{2} d x \leq C \int_{B_{r}} w^{\gamma / 4+1}|\nabla \eta|^{2} d x .
$$

By the Sobolev embedding theorem, (2.17) yields

$$
\left(r^{-m} \int_{B_{\gamma / 2}} w^{(\gamma / 2+1) \frac{m}{m-2}} d x\right)^{\frac{m-2}{m}} \leq C r^{-m} \int_{B_{r}} w^{\gamma / 4+1} d x
$$


Put $1+\varepsilon:=\left(1+\frac{\gamma}{2}\right) \frac{m}{m-2}$, then we obtain the inequality

$$
\left(r^{-m} \int_{B_{r / 2}} e^{(1+\varepsilon)|\nabla u|^{2}} d x\right)^{\frac{m-2}{m}} C r^{-m} \int_{B_{r}} e^{|\nabla u|^{2}} d x \leq C r^{-m} E_{0},
$$

and $1+\gamma / 4=\frac{(1+\varepsilon)(m-2)+1}{2 m}$. Finally we remark that we can not take $\gamma=0$.

(2.18) Proposition. Assume $m \geq 3$. Let $u$ be a minimizer of $E$. For a finite number $q$ satisfying $q>\frac{m}{2}$, if $B_{r}(a) \subset \Omega$, then the following inequality holds:

$$
\left(r^{-m} \int_{B_{r / 2}(a)} e^{q|\nabla u|^{2}} d x\right)^{\frac{1}{q}} \leq C,
$$

where $C=C\left(m, E_{0}, d, q\right)$.

Proof. In (2.6), if we take $\gamma \geq 0$, then the first and second terms are estimated by

$$
(\mathrm{I}+\mathrm{II}) \geq\left.\left.\frac{1}{2}\left(1+\frac{\gamma}{2}\right) \int_{B_{r}}|\nabla| \nabla u\right|^{2}\right|^{2} w^{\gamma / 2+1} \eta^{2} d x
$$

Hence we have

$$
\int_{B_{\gamma}}\left|\nabla\left(w^{\frac{1}{2}(\gamma / 2+1)}\right)\right|^{2} \eta^{2} d x \leq \frac{C}{\delta} \int_{B_{\gamma}}\left|\nabla\left(w^{\frac{1}{2}(\gamma / 2+1)}\right)\right| w^{\frac{1}{2}(\gamma / 2+1)+\delta}|\nabla \eta| \eta d x
$$

for any $\delta>0$, where the constant $C=C(\gamma)$.

Take $\alpha=\gamma / 2+1$. Then using the Young's inequality and the Sobolev embedding theorem, (2.19) yields that

$$
\left(r^{-m} \int_{B_{\sigma r}} w^{\alpha \frac{m}{m-2}} d x\right)^{\frac{m-2}{m}} \leq \frac{C r^{-m}}{(1-\sigma)^{2}} \int_{B_{r}} w^{\alpha+\delta} d x,
$$

where $C=C(\delta, \gamma)$.

Taking $\alpha=1$ and $\delta=\varepsilon$, by Proposition 2.10 and (2.20), we have

$$
\begin{aligned}
\left(r^{-m} \int_{B_{\sigma^{2} r}} e^{\frac{m}{m-2}|\nabla u|^{2}} d x\right)^{\frac{m-2}{m}} & \leq \frac{C}{(1-\sigma)^{2}} r^{-m} \int_{B_{\sigma r}} e^{(1+\varepsilon)|\nabla u|^{2}} d x \\
& \leq\left(\frac{C}{(1-\sigma)^{2}}\right)^{2}\left(r^{-m} E_{0}\right)^{\frac{m}{m-2}} .
\end{aligned}
$$


In the following, take $\alpha=\left(\frac{m}{m-2}\right)^{(k-1) / 2}$ and $\delta=\left(\frac{m}{m-2}\right)^{k / 2}-\left(\frac{m}{m-2}\right)^{(k-1) / 2}$

Then finite times iterations of this procedure implies that

$$
\left(r^{-m} \int_{B_{\sigma} k_{r}} e^{\left(\frac{m}{m-2}\right)^{k / 2}|\nabla u|^{2}} d x\right)^{\left(\frac{m-2}{m}\right)^{k / 2}} \leq C\left(r^{-m} E_{0}\right)^{\left(\frac{m}{m-2}\right)^{k / 2}},
$$

where the constant $C=(m, k, \sigma, \gamma)$. Therefore for any finite number $q$ satisfying $q>\frac{m}{2}$ the following inequality is true:

$$
\left(r^{-m} \int_{B_{r / 2}} e^{q|\nabla u|^{2}} d x\right)^{1 / q} \leq C
$$

(2.22) Proposition. Assume $m \geq 3$. Let $u$ be a minimizer of $E$. If $B_{2 r}(a) \subset \Omega$, then there exists a positive number $p$ satisfying $1<p<\frac{m}{m-2}$ such that for $\alpha \geq 2$ and $0<\sigma<1$,

$$
\left(r^{-m} \int_{B_{\sigma r}(a)}\left(1+|\nabla u|^{2}\right)^{\alpha \frac{m}{m-2}} d x\right)^{\frac{m-2}{m}} \leq C\left(r^{-m} \int_{B_{r}(a)}\left(1+|\nabla u|^{2}\right)^{\alpha p} d x\right)^{1 / p},
$$

where the constant $C=C\left(m, d, E_{0}, \sigma, \alpha, p\right)$.

Proof. In (2.6), replace $\varphi$ to $\nabla_{k} \varphi$, set $w:=1+|\nabla u|^{2}$ and substitute $\varphi:=\nabla_{k} u^{\alpha} w^{\gamma / 2} \eta^{2}$ for $\gamma \geq 0$, then we have

$$
\begin{aligned}
& \int_{B_{r}}\left(\nabla_{k} \nabla_{i} u^{\alpha}+\nabla_{i} u^{\alpha} \nabla_{k}|\nabla u|^{2}\right) \nabla_{k} \nabla_{i} u^{\alpha} w^{\gamma / 2} \eta^{2} e^{|\nabla u|^{2}} d x \\
& +\frac{\gamma}{2} \int_{B_{r}}\left(\nabla_{k} \nabla_{i} u^{\alpha}+\nabla_{i} u^{\alpha} \nabla_{k}|\nabla u|^{2}\right) \nabla_{k} u^{\alpha} \nabla_{i} w w^{\gamma / 2-1} \eta^{2} e^{|\nabla u|^{2}} d x \\
= & -2 \int_{B_{r}}\left(\nabla_{k} \nabla_{i} u^{\alpha}+\nabla_{i} u^{\alpha} \nabla_{k}|\nabla u|^{2}\right) \nabla_{k} u^{\alpha} w^{\gamma / 2} \nabla_{i} \eta \eta e^{|\nabla u|^{2}} d x .
\end{aligned}
$$

By a similar calculation to the proof of Proposition 2.10, we have

$$
\begin{aligned}
\text { the first term of }(2.22)= & \int_{B_{r}}\left|\nabla^{2} u\right|^{2} w^{r / 2} \eta^{2} e^{|\nabla u|^{2}} d x \\
& +\left.\left.\int_{B_{r}}|\nabla| \nabla u\right|^{2}\right|^{2} w^{\gamma / 2} \eta^{2} e^{|\nabla u|^{2}} d x, \\
\text { the second term of }(2.22)= & \int_{B_{r}}\left|\left\langle\nabla u, \nabla|\nabla u|^{2}\right\rangle\right|^{2} w^{\gamma / 2-1} \eta^{2} e^{|\nabla u|^{2}} d x
\end{aligned}
$$




$$
\begin{gathered}
+\left.\left.\int_{B_{r}}|\nabla| \nabla u\right|^{2}\right|^{2} w^{\gamma / 2-1} \eta^{2} e^{|\nabla u|^{2}} d x, \\
\mid \text { the third term of (2.22)| } \leq 2 \int_{B_{r}}|\nabla w||\nabla \eta| w^{\gamma / 2+1} \eta e^{|\nabla u|^{2}} d x \\
\leq C \int_{B_{r}}\left|\nabla w^{\frac{\gamma+4}{4}}\right|^{2} \eta^{2} e^{|\nabla u|^{2}} d x \\
+\frac{1}{2} \int_{B_{r}} w^{\frac{\gamma+4}{2}}|\nabla \eta|^{2} e^{|\nabla u|^{2}} d x .
\end{gathered}
$$

Therefore we have

$$
\int_{B_{r}}\left|\nabla\left(\eta w^{\frac{\gamma+4}{4}}\right)\right|^{2} d x \leq C \int_{B_{r}} w^{\frac{\gamma+4}{2}}|\nabla \eta|^{2} e^{|\nabla u|^{2}} d x .
$$

By the Sobolev embedding theorem, we obtain

$$
\left(r^{-m} \int_{B_{\sigma r}} w^{\frac{r+4}{2} \frac{m}{m-2}} d x\right)^{\frac{m-2}{m}} \leq C r^{-m} \int_{B_{r}} w^{\frac{r+4}{2}} e^{|\nabla u|^{2}} d x .
$$

Applying the Hölder's inequality to (2.23), we obtain the inequality

$$
\left(r^{-m} \int_{B_{\sigma r}} w^{\frac{\gamma+4}{2} \frac{m}{m-2}} d x\right)^{\frac{m-2}{m}} \leq C\left(r^{-m} \int_{B_{r}} w^{p^{\frac{\gamma+4}{2}}} d x\right)^{1 / p}\left(r^{-m} \int_{B_{r}} e^{q|\nabla u|^{2}} d x\right)^{1 / q} .
$$

On the other hand, choose $q>\frac{m}{2}$ then $1<p<\frac{m}{m-2}$ and the integral $r^{-m} \int_{B_{r}} e^{q|\nabla u|^{2}} d x$ is bounded from above. Therefore, setting $\alpha=\frac{r+4}{2} \geq 2$, we complete the proof.

(2.24) Proposition. Assume $m \geq 3$. Let $u$ be a minimizer of $E$. If $B_{2 r}(a) \subset \Omega$, then there exists a constant $C=C\left(m, d, E_{0}\right)$ such that

$$
\sup _{B_{r / 2}(a)}|\nabla u|^{2} \leq C \text {. }
$$

Proof. By Proposition 2.22, there exists a positive number $p$ satisfying $p<\frac{m}{m-2}$ such that for any $\alpha \geq 2$ and any $0<\sigma<1$

$$
\left(r^{-m} \int_{B_{\sigma r}} v^{\alpha \frac{m}{m-2}} d x\right)^{\frac{m-2}{m}} \leq C\left(r^{-m} \int_{B_{r}} v^{\alpha p} d x\right)^{1 / p},
$$

where $v:=1+|\nabla u|^{2}$. Take 


$$
\begin{gathered}
r_{k}:=\sigma^{k} r, \quad B_{k}:=B_{r_{k}}, \\
\alpha_{k}:=2\left(\frac{1}{p} \frac{m}{m-2}\right)^{k} .
\end{gathered}
$$

Then we obtain a recursion formula

$$
\left(r^{-m} \int_{B_{k}} v^{\alpha_{k} \frac{m}{m-2}} d x\right)^{\alpha_{k}^{-1} \frac{m-2}{m}} \leq C\left(r^{-m} \int_{B_{k-1}} v^{\alpha_{k-1}} d x\right)^{\alpha_{k-1}^{-1}} \text { for } k \geq 1
$$

Therefore it holds that

$$
\left(r^{-m} \int_{B_{k}} v^{\alpha_{k} \frac{m}{m-2}} d x\right)^{\alpha_{k}^{-1} \frac{m-2}{m}} \leq C\left(r^{-m} \int_{B_{0}} v^{p} d x\right)^{1 / p} \text { for } k \geq 1 .
$$

Letting $k \rightarrow \infty$, we have

$$
\sup _{B_{\gamma / 2}} v \leq C\left(r^{-m} \int_{B_{r}} v^{p} d x\right)^{1 / p} .
$$

Therefore

$$
\begin{aligned}
\sup _{B_{r / 2}}|\nabla u|^{2} & \leq C\left(r^{-m} \int_{B_{r}}\left(1+|\nabla u|^{2}\right)^{p} d x\right)^{1 / p} \\
& \leq C\left(r^{-m} \int_{B_{r}} e^{|\nabla u|^{2}} d x\right)^{1 / p} \leq C\left(r^{-m} E_{0}\right)^{1 / p} .
\end{aligned}
$$

(2.25) Remark. In the case of $m=2$, the argument similar to the proof of Proposition 2.10 yields

$$
\left(r^{-2} \int_{B_{r / 2}(a)} e^{(1+\varepsilon)|\nabla u|^{2}} d x\right)^{\frac{2-q}{q}} \leq C r^{-2} \int_{B_{\gamma}(a)} e^{|\nabla u|^{2}} d x,
$$

for any $1<q<2$ and $0<\varepsilon<\varepsilon_{0}$, where the constant $C=C(\varepsilon,|\Omega|)$. The inequality $\left(2.10^{\prime}\right)$ is proved by taking $\gamma$ as $\frac{1}{4}(1 / q-1)<\gamma<0$ in the proof of Proposition 2.10.

We also obtain inequalities similar to (2.18) and (2.22). Therefore Proposition 2.24 also holds in case of $m=2$.

\section{Hölder continuity of a minimizer}

In the final section, we shall prove the main theorem. The proof of the 
theorem is divided into several propositions. Using the bound of the gradient of minimizers, we shall obtain an estimate of the growth order and the mean value of them on a small ball.

(3.1) Proposition. For $0<r<R_{0} / 4$ satisfying $B_{R_{0}}(a) \subset \Omega$ and any constant vector $V \in \mathbf{R}^{n m}$ satisfying

$$
|V| \leq \sup _{B_{R_{0}}(a)}|\nabla u|,
$$

there exists a constant vector $V_{\sigma} \in \mathbf{R}^{n m}$ such that

$$
\begin{gathered}
\left|V_{\sigma}\right| \leq \sup _{B_{R_{0}}(a)}|\nabla u|, \\
\int_{B_{\sigma r}(a)}\left|\nabla u-V_{\sigma}\right|^{2} d x \\
\leq C \sigma^{m+2} \int_{B_{r^{\prime}}(a)}|\nabla u-V|^{2} d x+C \sigma^{m} \int_{B_{r^{\prime}}(a)}|\nabla u-V|^{2} d x,
\end{gathered}
$$

for all $0<\sigma<1 / 2$.

Proof. Let $v: B_{r} \rightarrow \mathbf{R}^{n}$ be an $H^{1}$-solution of $\Delta v=0$ with $u=v$ on $B_{r}$. By the definition of $v$, for any $\varphi \in C_{0}^{\infty}\left(B_{r}\right)$, we have

$$
\int_{B_{\gamma}} \nabla_{i} v^{\alpha} \nabla_{i} \varphi^{\alpha} e^{|V|^{2}} d x=0 .
$$

Set $V_{\sigma}:=\left(\operatorname{vol}\left(B_{\sigma r}\right)\right)^{-1} \int_{B_{\sigma r}} \nabla v d x$. By applying the maximum principle and some well known results on the Laplace equation, we obtain

$$
\begin{aligned}
\left|V_{\sigma}\right| & \leq\left(\operatorname{vol}\left(B_{\sigma r}\right)\right)^{-1} \int_{B_{\sigma r}}|\nabla v| d x \\
& \leq \sup _{B \sigma r}|\nabla v| \leq \sup _{\partial B_{r}}|\nabla v| \leq \sup _{\partial B_{r}}|\nabla u| \leq \sup _{B_{R_{0}}}|\nabla u| .
\end{aligned}
$$

Therefore we conclude (3.2).

On the other hand, we have

$$
\int_{B_{\sigma r}}\left|\nabla u-V_{\sigma}\right|^{2} d x \leq 2 \int_{B_{\sigma r}}|\nabla u-\nabla v|^{2} d x+2 \int_{B_{\sigma r}}\left|\nabla v-V_{\sigma}\right|^{2} d x
$$

since $\left|\nabla u-V_{\sigma}\right|^{2} \leq 2|\nabla u-\nabla v|^{2}+2\left|\nabla v-V_{\sigma}\right|^{2}$. Here we note that $v$ satisfies a strongly elliptic system with constant coefficients: $\Delta v=0$. Therefore $v$ satisfies 
an estimate due to Campanato [4, III(2.8) or (2.11)], namely

$$
\int_{B_{\sigma r}}\left|\nabla v-V_{\sigma}\right|^{2} d x \leq C \sigma^{m+2} \int_{B_{r}}\left|\nabla v-V_{1}\right|^{2} d x .
$$

Since $V_{1}$ minimizes the integral $\int_{B_{r}}|\nabla v-c|^{2} d x$, we have

$$
\int_{B_{r}}\left|\nabla v-V_{1}\right|^{2} d x \leq \int_{B_{r}}|\nabla v-V|^{2} d x
$$

Combining (3.5) and (3.6), we obtain

$$
\begin{aligned}
& \int_{B_{\sigma r}}\left|\nabla v-V_{\sigma}\right|^{2} d x \leq C \sigma^{m+2} \int_{B_{r}}|\nabla v-V|^{2} d x \\
& \leq C \sigma^{m+2} \int_{B_{r}}|\nabla u-\nabla v|^{2} d x+C \sigma^{m+2} \int_{B_{r}}|\nabla u-V|^{2} d x .
\end{aligned}
$$

In order to estimate $\int_{B_{r}}|\nabla u-\nabla v|^{2} d x$, we use the function $\varphi=u-v$ defined on $B_{r}$. From differential equations, we derive

$$
\begin{gathered}
\int_{B_{r}} \nabla_{i} u^{\alpha} \nabla_{\imath} \varphi^{\alpha}\left[\int_{0}^{1} \partial_{t}\left(e^{|t V+(1-t) \nabla u|^{2}}\right) d t\right] d x \\
-\int_{B_{r}} \nabla_{\imath} u^{\alpha} \nabla_{i} \varphi^{\alpha} e^{|V|^{2}} d x+\int_{B_{r}} \nabla_{i} v^{\alpha} \nabla_{i} \varphi^{\alpha} e^{|V|^{2}} d x=0 .
\end{gathered}
$$

Since

$$
\partial_{t}\left(e^{|t V+(1-t) \nabla u|^{2}}\right)=2\left(V_{k}^{\beta}-\nabla_{k} u^{\beta}\right)\left(t V_{k}^{\beta}+(1-t) \nabla_{k} u^{\beta}\right) e^{|t V+(1-t) \nabla u|^{2}},
$$

we have

$$
\begin{aligned}
& \int_{B_{r}}|\nabla u-\nabla v|^{2} d x \leq 2 \int_{B_{r}} \int_{0}^{1}|\nabla u||\nabla u-\nabla v||\nabla u-V| \\
& \times|t V+(1-t) \nabla u| e^{|t V+(1-t) \nabla u|^{2}} d t d x .
\end{aligned}
$$

Since $|V| \leq \sup _{B_{R_{0}}}|\nabla u|$ and $|t V+(1-t) \nabla u| \leq|V|+|\nabla u|$, using Proposition 3.17 , we have

$$
\text { (the left hand side) } \leq C \int_{B_{r}}|\nabla u-\nabla v||\nabla u-V| d x \text {. }
$$

Therefore we conclude 


$$
\int_{B_{r}}|\nabla u-\nabla v|^{2} d x \leq C \int_{B_{r}}|\nabla u-V|^{2} d x
$$

where the constant $C=C\left(m, d, E_{0}\right)$.

A calculation similar to lead (3.8) also yields that

$$
\int_{B_{\sigma r}}|\nabla u-\nabla v|^{2} d x \leq C \int_{B_{2 \sigma r}}|\nabla u-V|^{2} d x
$$

On the other hand, since $\tilde{u}^{\alpha}:=u^{\alpha}+\sum_{i=1}^{\mathrm{m}} V_{i}^{\alpha} x^{i}$ is also a weak solution of the Euler-Lagrange equation of $E$, if $\sigma<\frac{1}{2}$, then we have

$$
\int_{B_{2 \sigma r}}|\nabla u-V|^{2} d x \leq C \sigma^{m} \int_{B_{r}}|\nabla u-V|^{2} d x .
$$

Combining (3.4), (3.7), (3.8) and (3.9), we derive

$$
\int_{B_{\sigma r}}\left|\nabla u-V_{\sigma}\right|^{2} d x \leq C \sigma^{m+2} \int_{B_{r}}|\nabla u-V|^{2} d x+C \sigma^{m} \int_{B_{r}}|\nabla u-V|^{2} d x .
$$

(3.10) Proposition. Let $U_{r}(a)=U_{r} \in \mathbf{R}^{n m}$ be the average of $\nabla u$ in $B_{r}(a)$, i.e,

$$
U_{r}(a)=\left(\operatorname{vol}\left(B_{r}(a)\right)\right)^{-1} \int_{B_{r}(a)} \nabla u d x
$$

If $B_{R_{0}}(a) \subset \Omega$ and $0<r<R_{0} / 4$, then there exists a constant $\mu$ satisfying $0<\mu<1$ such that

$$
\int_{B_{r}(a)}\left|\nabla u-U_{r}\right|^{2} d x \leq C r^{m+2 \mu}
$$

where the constant $C=C\left(m, d, E_{0}\right)$.

Proof. By Proposition 3.1, we may choose a number $\sigma \in(0,1 / 2)$ such that the following is true; for $r_{i}:=\sigma^{i} \cdot r$ and $B_{\imath}:=B_{r_{i}}$, there exist constant vectors $W_{0}, W_{1}, \ldots, W_{k}$ such that

$$
r_{i+1}^{-m} \int_{B_{i+1}}\left|\nabla u-W_{i+1}\right|^{2} d x \leq C \sigma^{2} r_{i}^{-m} \int_{B_{i}}\left|\nabla u-W_{i}\right|^{2} d x+C \int_{B_{i}}\left|\nabla u-W_{i}\right|^{2} d x
$$




$$
\leq C_{0} \sigma^{2} r_{i}^{-m} \int_{B_{i}}\left|\nabla u-W_{i}\right|^{2} d x+C_{1}
$$

provided $\left|W_{i}\right| \leq \sup _{B_{R_{0}}}|\nabla u|^{2}$, where the constant $C_{1}$ depends on the bound of $|\nabla u|$ on $B_{R_{0}}$. By Proposition 3.1 again, (3.11)-(3.13) remain valid for all positive integers $i$. Therefore choose $\sigma$ so small that $\sigma$ satisfies $C_{0} \sigma^{2}<1$. Then (3.13) shows that there exist constants $C$ and $\mu$ satisfying $0<\mu<1$ depending on $m, d, E_{0}$ such that

$$
r^{-(m+2 \mu)} \int_{B_{r}}\left|\nabla u-U_{r}\right|^{2} d x \leq C .
$$

Proposition 3.10 implies that if $B_{4 r}(a) \subset \Omega$ then $\nabla u$ is Hölder continuous on $B_{r}(a)$ with the exponent $\mu$.

Acknowledgement. This research was done while the author visited the Mathematics Research Center, University of Warwick at the occasion of the Symposium on "Partial Differential Equations in Geometry". He gives special thanks the organizers of the symposium and the Mathematics Institute of the University of Warwick for their kindness. In particular, he would like to thank Professor J. Eells for suggesting him this research and for his continuous encouragement, and Professor M. Micallef and Professor P. Avilés for their encouragements and kindness. Moreover, he thanks Professor K. Tanaka in Nagoya University, who read the first manuscript of this research and gave him valuable advices.

The author also would like to thank Professor J. Eells and Professor L. Lemaire. They read the first manuscript of this research and point out author's mistakes. Finally, he thanks Professor S. Bando and Professor S. Nishikawa who give him valuable comments.

\section{REFERENCES}

[1] E. DiBenedetto, $C^{1+\alpha}$ local regularity of weak solutions of degenerate elliptic equations, Nonlinear Analysis: TMA, 7 (1983), 827-850.

[2] D. M. Duc and J. Eells, Regularity of exponentially harmonic function, to appear in Internat. J. Math.,

[ 3 ] J. Eells and L. Lemaire, Some properties of exponential harmonic maps, preprint.

[4] M. Giaquinta, "Multiple Integrals in the Calculus of Variations and Nonlinear Analysis," Princeton Univ. Press, Princeton, New Jersey, 1983.

[5] M. Giaquinta and E. Giusti, On the regularity of the minima of variational integ. rals, Acta Math., 148 (1982), 31-46.

[6] D. Gilberg and N. S. Trudinger, "Elliptic Partial Differential Equations of Second 
Order, Second edition," Springer-Verlag, Berlin-Heiderberg-New York, 1983.

[ 7 ] R. Hardt and F. H. Lin, Mapping minimizing the $L^{p}$-norm of the gradient, Comm. Pure and Appl. Math., 40 (1987), 555-588.

[8] K. Masuda, "Nonlinear Mathematics," Asakura, Tokyo, 1985. (in Japanese)

[9] C. B. Morrey, "Multiple Integrals in the Calculus of Variations," Springer-Verlag, Berlin-Heiderberg-New York, 1966.

[10] R. Schoen and K. Uhlenbeck, A regularity theory for harmonic maps, J. Differential Geom., 18 (1982), 307-335.

[11] P. Tolksdorff, Everywhere regularity for some quasi-linear system with a lack of ellipticity, Annali di Math. Pure et Applicata, 134 (1983), 241-266.

[12] K. Uhlenbeck, Regularity of nonlinear elliptic systems, Acta Math., 138 (1977), $219-240$.

[13] B. White, Homotopy classes in Sobolev spaces and the existence of energy minimizing maps, Acta Math., 160 (1988), 1-17.

Department of Mathematics

School of Science

Nagoya University

Nagoya 464-01, JAPAN 